\title{
The Gut in Heart Failure: Current Knowledge and Novel Frontiers
}

\author{
Antonella Gallo $^{\mathrm{a}}$ Noemi Macerola ${ }^{\mathrm{a}}$ Angela Maria Favuzzi ${ }^{\mathrm{a}}$ \\ Maria Anna Nicolazzi ${ }^{a}$ Antonio Gasbarrini ${ }^{a}{ }^{a}$ Massimo Montalto ${ }^{a, b}$ \\ aDepartment of Medical Sciences, Fondazione Policlinico Universitario A. Gemelli IRCCS, Rome, Italy; \\ bUniversità Cattolica del Sacro Cuore, Rome, Italy
}

\section{Highlights of the Study}

- The gut plays a relevant pathophysiological role in heart failure (HF).

- The interplay between the cardiac and intestinal systems is driven by different pathways.

- Modulation of the intestinal microflora may represent a potential treatment for HF.

- Dysbiosis reflects in the upregulation of sodium/hydrogen cotransporters with a consequent overload of salt and fluids. Their inhibition may represent a novel strategy.

\section{Keywords}

Gut microbiota - Dysbiosis · Heart failure - Trimethylamine $\mathrm{N}$-oxide $\cdot$ Short-chain fatty acid $\cdot$ Sodium-hydrogen

exchanger 3

\begin{abstract}
Heart failure (HF) represents a major health problem affecting millions of people worldwide. In the latest years, many efforts have been made to search for more effective strategies to prevent and modify the course of this disease, but results are still not satisfying. HF represents a complex clinical syndrome involving many other systems, including the gastrointestinal system. Although the relationship between the gut and $\mathrm{HF}$ is far from being fully understood, based on recent evidence highlighting the putative role of the gastrointestinal system in different cardiovascular diseases, it is conceivable that the gut-heart link may represent the basis for novel therapeutic approaches in the HF context as well.
\end{abstract}

This intricate interplay involving typical hemodynamic changes and their consequences on gut morphology, permeability, and function, sets the stage for alterations in microbiota composition and is able to impact mechanisms of $\mathrm{HF}$ through different routes such as bacterial translocation and metabolic pathways. Thus, the modulation of the gut microbiota through diet, probiotics, and fecal transplantation has been suggested as a potential therapeutic approach. More interestingly, another effect of alteration in microbiota composition reflects in the upregulation of cotransporters (NHE3) with consequent salt and fluid overload and worsening visceral congestion. Therefore, the inhibitors of this cotransporter may also represent a novel therapeutic frontier. By review of recent data on this topic, we describe the current state of the complex interplay between the gastrointestinal and cardiac systems in $\mathrm{HF}$, and the relevance of this knowledge in seeking new therapeutic strategies.

(c) 2022 The Author(s).

Published by S. Karger AG, Basel
C 2022 The Author(s).

Published by S. Karger AG, Basel

This is an Open Access article licensed under the Creative Commons Attribution-NonCommercial-4.0 International License (CC BY-NC) (http://www.karger.com/Services/OpenAccessLicense), applicable to the online version of the article only. Usage and distribution for commercial purposes requires written permission.
Correspondence to:

Antonella Gallo, antonella.gallo@ policlinicogemelli.it 


\section{Introduction}

\section{The Gut-Heart Axis}

Cardiovascular diseases (CVDs) still represent the leading cause of death and disability in developed countries [1]. It is, therefore, obvious that more and more efforts have been made to search for effective strategies to prevent and modify the course of these diseases. It is well known that many factors contribute to the rise and progression of CVDs, from genetic or environmental sources, or a combination of both [2]. New knowledge on the pathophysiology of heart diseases, the roles of common risk factors, such as obesity and type 2 diabetes, and the interplay between cardiovascular and other systems represent the mainstay of research $[3,4]$.

Gastrointestinal and hepatic involvement in heart diseases has usually received little attention, and the mechanisms underlying the specific link between the gastrointestinal and cardiac systems are not completely understood. However, their bidirectional relationship has proven to be particularly close, thus leading to the identification of the so-called "gut-heart axis" [5]. In particular, much attention has been given to the contribution of intestinal microbiota in the etiology of CVDs. For years, the gut microbiota has been considered merely a part of the digestive system [6]. Nowadays, however, it is widely recognized as an integrated endocrine system, producing a large series of metabolism-dependent and metabolismindependent signals with a strong impact on CVDs [6]. It is known that the human body harbors at least $10^{14} \mathrm{mi}$ croorganisms belonging to $>2,000$ microbial species, colonizing its internal and external surfaces in an integrated ecosystem in which humans and microbes benefit each other with functions they have not evolved on their own $[7,8]$.

When in balance, this system provides us with vitamin synthesis, development of a healthy host immune system, metabolic functions by the production of short-chain fatty acids (SCFAs) involved in energy harvest and storage, and the trimethylamine $\mathrm{N}$-oxide (TMAO) pathway [8]. The maintenance of biodiversity is crucial to keep the system in balance and to guarantee homeostasis [9]. However, during life, the composition of gut microbiota varies considerably among individuals, being influenced by changes in environmental conditions (diet, various drugs, oxidative stress) or host genetic factors $[10,11]$.

The interplay between gut microbiota and host cardiometabolic functions has been investigated in recent times, mainly the role of TMAO in CVDs [12-19]. The generation of TMAO by multiple dietary nutrients and their blood levels is modulated by intestinal microbiota. Foods rich in cholesterol and fats are also rich in lecithin, choline, and L-carnitine. After ingestion, the gut microbiota can cleave the $\mathrm{C}-\mathrm{N}$ bond of these nutrients by the enzyme TMA lyase, releasing intermediate trimethylamine (TMA) as a waste product. The subsequent hepatic oxidation via flavin monooxygenase (particularly flavin monooxygenase 3) leads to the production of TMAO entering the circulation [12-14]. Elevated concentrations of TMAO are widely correlated with the accumulation of cholesterol in macrophages and the development of atherosclerosis; several studies have confirmed the association between increased levels of TMAO and higher incidence of cardiovascular events in coronary artery disease [12-19]. In particular, the association of blood levels of TMAO (and its precursors) with the development of major adverse cardiovascular events was recognized to be dose-dependent and independent of conventional risk factors such as kidney dysfunction, diabetes mellitus, and obesity [20].

Among CVDs, heart failure (HF) represents a major global health problem, being a leading cause of death and morbidity worldwide [21]. Recent progress in understanding the pathophysiology of HF leads to the investigation of new approaches, although results are still far from being satisfactory [3]. In this regard, the potential role of the gut in the pathophysiology of HF has recently received increased attention. Besides bacterial metabolites such as TMAO in this condition, more complex interactions involving hemodynamic changes and their consequences on gut morphology, permeability, and function contribute to maintain a deleterious vicious cycle, worthy of being explored $[4,5,22,23]$. In this review, we describe the current state of the relationship between the gastrointestinal system and HF and the relevance of these understandings in seeking new therapeutic strategies.

\section{The "Gut Hypothesis" of HF}

HF represents a complex clinical syndrome, characterized by typical symptoms (breathlessness and fatigue), that may be accompanied by signs such as elevated jugular venous pressure, pulmonary crackles, peripheral edema, and ankle swelling [21]. As widely reported, patients with HF are very heterogeneous [24]. More than $70 \%$ of patients with HF suffer from one or more comorbidities, such as type 2 diabetes mellitus, chronic kidney disease, chronic obstructive pulmonary disease, etc., that contrib- 
ute to disease progression and have an independent effect on mortality [25].

Classification of HF is usually based on left ventricular (LV) ejection fraction (EF), considering values of $\geq 50 \%$ as the threshold to identify patients affected by HF with a preserved EF, and by New York Heart Association (NYHA) functional classification based on clinical picture [21]. Although HFpEF has been previously considered as the precursor of HF with a reduced EF (HFrEF), it is now widely accepted that these represent two different disorders. In particular, HFpEF is usually associated with comorbidities, such as obesity, metabolic syndrome, and diabetes mellitus [26], and its incidence has been growing significantly in recent years due to aging and increased burden of comorbidities of the general population.

Clinical signs and biomarkers have a fundamental diagnostic and prognostic role in HF. The most important biomarker is B-type natriuretic peptide (BNP), usually related to increased LV filling pressures and wall stress. Its high diagnostic negative predictive value and close correlation with response to therapy have made it a very useful parameter to guide pharmacological strategies [21]. However, levels of BNP are usually lower in patients with HFpEF than in those with HFrEF, and this may be influenced by sex and comorbidities; for example, obesity is associated with reduced natriuretic peptide levels [27].

However, new biomarkers have attracted increasing interest because of emerging understanding on the complex pathophysiological background of HF, including the central role of low-grade inflammation [28-32]. Preliminary studies have reported increased plasma levels of proinflammatory cytokines, including interleukin 6 (IL-6), tumor necrosis factor (TNFa), galectin-3, and C-reactive protein (CRP) in HF patients, also showing a correlation with a worse prognosis [33].

Currently, treatments are based mainly on neuroendocrine inhibition by angiotensin-converting enzyme inhibitors and angiotensin receptor blockers, aldosterone antagonists, beta-blockers, combination of angiotensin receptor blockers/neprilysin blockers, and ivabradine [21]. More recently, the PARADIGMHF trial showed that sacubitril/valsartan was superior to enalapril in reducing the risks of death and hospitalization for HFrEF [34]. However, despite these cardioprotective therapeutic options, mortality and hospitalization rates remain high and quality of life poor, thus encouraging active research in this field.

The Interplay between Intestinal and Cardiac Systems in HF
Recently, attention was focused on the pathophysiologic role of some comorbidities, such as anemia and insulin resistance, with the aim to improve knowledge of the pathways involved in cardiac disease progression and to identify novel drug targets, mainly for those patients with HFpEF [35]. In diabetic patients, sodium-glucose cotransporter 2 inhibitors (empagliflozin, canagliflozin, and dapagliflozin) have consistently shown a reduced risk of HF hospitalization or CV death, probably by a direct pleiotropic effect on cardiomyocytes, even if the exact mechanism of action is largely unknown [35].

Tang's group highlighted the potential pathogenetic role of the gut in HF, formulating the first statement of the "gut hypothesis of HF" [22]. This relationship has aroused a great deal of interest. As described in the following paragraphs, both venous congestion and reduced cardiac output in HF induce bowel hypoperfusion, leading to mucosal ischemia and hypoxia of the villous structure of the intestinal wall. This impairment in intestinal barrier function increases bowel wall permeability, leading to increased bacterial translocation into the circulatory system and increased circulating endotoxins, thus promoting a typical inflammatory state [23]. Here, we will report current understanding about the main physiopathologic mechanisms holding the complex relationship between gut and HF.

\section{Altered Gut Circulation and HF}

An important aspect in gut-derived inflammation in patients with HF is the altered gut circulation as a consequence of reduced cardiac output with consequent sympathetic vasoconstriction, leading to redistribution of the systemic circulation and venous congestion in multiple organs, including the bowel wall.

The microstructure of the intestinal villus surface not only presents an ideal structure to optimize nutrient absorption but also makes it susceptible to shunting of oxygenated blood through the base of the villus, putting the villus tip at risk of a relative ischemia [36]. The descending gradient of tissue oxygen concentration from the base to the tip of the villus is inversely related to blood flow, and it is directly influenced by alterations in perfusion [23]. Patients with chronic HF show a thickened bowel wall, suggestive of bowel wall edema, dysfunction of the intestinal barrier, and significant increased intestinal permeability compared to control patients [37]. Sandek et al. [38] found in $65 \mathrm{HF}$ patients a significant reduction in systolic blood flow (assessed by ultrasound) in the supe-

Med Princ Pract 2022;31:203-214 205 
rior and inferior mesenteric arteries and celiac trunk compared with 25 controls, with a significant correlation between impaired intestinal blood flow and severity of HF. Moreover, patients with lower celiac trunk blood flow showed higher thickness of the bowel wall, suggestive of bowel wall edema, more abdominal discomfort, and increased concentration of juxtamucosal anaerobic bacteria in the sigmoid colon, correlating with higher systemic levels of IgA anti-lipopolysaccharide (LPS). It has been suggested that the interaction between mucosal intestinal bacteria and the immune system may contribute to the systemic inflammation in HF [38]. Impaired intestinal blood flow associated with hepatosplenic congestion and, consequently with intestinal barrier dysfunction, is also likely to determine a reduction of total absorptive surface area, representing one of the major determinants of the plasma profile of orally administered drugs [39].

Regardless of the underlying LV systolic function, from 20 to $60 \%$ of patients with HF can develop right ventricular (RV) dysfunction [40]. Patients with systolic RV dysfunction have a pressure overload which is transmitted to the venous system. Venous congestion has a prognostic value in risk of HF but is also an integral part of pathogenesis of HF and of other organ dysfunctions, since it contributes to worsening of renal function, as an example [41]. Valentova et al. [42] demonstrated that splanchnic congestion was a typical finding in cachectic patients affected by HF with RV dysfunction. In $165 \mathrm{HF}$ patients with an LV EF $\leq 40 \%$, the combination of RV dysfunction and elevated right atrial pressure provided the best discrimination between cachectic and noncachectic patients. In particular, cardiac cachexia was associated with intestinal congestion irrespective of HF stage. Moreover, patients with higher level of right atrial pressure showed an increased bowel wall thickness associated with an increase in liver enzymes and clinical symptoms such as appetite loss, postprandial fullness, and inflammation [42]. It has been supposed that the edematous decompensation favors the translocation of LPS into the systemic circulation, perpetuating and worsening cachexia with the production of pro-inflammatory cytokines. As previously reported, ischemic-reperfusion damage to the gastrointestinal mucosa during cardiopulmonary bypass leads to significantly increased permeability with subsequent translocation into the bloodstream of intestinal gram-negative aerobic bacteria, which persists for the entire first perioperative period [43]. Circulating endotoxins (mainly LPS and peptidoglycans) exert their deleterious effects interacting with the host mucosal surface cells mainly through the Toll-like receptor 4 TLR4 pattern rec- ognition receptor and nucleotide oligomerization domain-containing receptors [28]. High levels of endotoxins promote the release of pro-inflammatory cytokines, specifically TNF- $\alpha$, IL- 1 , and IL-6, the recruitment of leukocyte subpopulations, endothelial cell adhesion molecule synthesis, and leucocyte integrin activation, ultimately leading to extravasation of inflammatory cells [29]. On the hemodynamic side, endotoxemia leads to subclinical systemic vasodilation, which is often associated with acute decompensated HF [36].

In patients with decompensated HF, LPS levels are higher in blood samples derived from the hepatic vein compared to the systemic circulation, thus confirming the hypothesis that LPS can be translocated from the gut [30]. Also increased intestinal permeability has been strictly correlated with the clinical severity of HF. Pasini et al. [31] studied 60 patients in stable condition with mild chronic HF (NYHA functional class I-II) and moderate to severe chronic HF (NYHA functional class III-IV); compared with normal control subjects, the entire chronic HF population showed significantly increased intestinal permeability (assessed by a cellobiose sugar test) and venous blood congestion (expressed as RAP by echocardiography), together with systemic inflammation parameters (CRP). Interestingly, these differences were more pronounced in patients with moderate to severe NYHA functional classes than in patients with the mild NYHA functional class. Moreover, intestinal permeability and levels of RAP and CRP were mutually interrelated [31].

It is well established that systemic inflammation plays a relevant prognostic role in the progression of HF by the chronic state of inflammation that accelerates atherosclerosis and cardiac fibrosis $[29,32]$. In particular, the biomarker substudy of the large sample "Acute Study of Clinical Effectiveness of Nesiritide and Decompensated Heart Failure" trial (ASCEND-HF trial) measured hsCRP levels at admission, at $48-72 \mathrm{~h}$, and 30 days later, showing that levels of hsCRP were considerably elevated at admission and after $48-72 \mathrm{~h}$, declining only at 30 days. In addition, increasing levels at 30 days after discharge are associated with higher 180-day mortality [32].

\section{The Alteration of Fluid Balance: The Role of NHE3}

When in balance, the GI tract represents a tightly controlled system which mediates the reabsorption of several liters of fluids on a daily basis. The disruption of adsorptive functions due to vascular impairment may cause a perturbation of fluid balance and an alteration of water 
homeostasis. Sodium balance is maintained by different groups of ion channels. Absorption of intestinal salt $(\mathrm{NaCl})$ and water in the gut are largely driven by the sodium-dependent cotransporters and sodium re-uptake, including the sodium-proton exchangers NHE2, NHE3, and NHE8, of which NHE3 is the most important [44]. NHE3 acts on the apical membrane of enterocytes, regulating the electroneutral exchange of sodium $(\mathrm{Na}+)$ and protons $(\mathrm{H}+)$, and plays a relevant role in transepithelial absorption of $\mathrm{Na}+$ and water [45]. It, therefore, represents a crucial element in maintaining a correct salt, volume, and acid-base homeostasis. Several factors, such as aldosterone, glucocorticoids, and increased concentrations of hydrogen $(\mathrm{H}+)$ can upregulate the function of this channel [46].

Ischemia deriving from venous congestion and vascular impairment generates a condition of hypoxia and a consequent cytoplasmic acidification in enterocytes, which leads to the upregulation of NHE3; this causes an increased reabsorption of sodium and simultaneous absorption of fluids, delivering a salt and water load on the cardiovascular system and worsening heart dysfunction. Moreover, the extrusion of $\mathrm{H}+$ ions into the gut lumen causes acidification of the local $\mathrm{pH}$, thus affecting the composition of the normal gut microbiota [47].

\section{Human Gut Microbiota and HF}

\section{Gut Microbiota Dysbiosis and HF}

Normal composition of gut microbiota consists mainly of bacteria from the phyla Bacteroidetes, Firmicutes, Actinobacteria, and to a lesser extent Proteobacteria [48]; among them, two phyla are dominant: Bacteroidetes (Bacteroides, Prevotella, and Xylanibacter) and Firmicutes (Ruminococcus, Clostridium, Lactobacillus, Eubacterium, Faecalibacterium, and Roseburia). Once the balance is broken, large shifts in the phyla of bacteria or expansion of new bacterial groups can take place; alteration of the ratio of Firmicutes/Bacteroidetes, reduction in microbial diversity, and outgrowth of Proteobacteria cause disequilibrium, which is the cardinal feature of this process [10, 11].

A growing number of diseases, including gastrointestinal diseases (IBDs, IBS, liver cirrhosis), extra-intestinal diseases (Parkinson's disease, major depression, autism spectrum disorder, chronic kidney disease), and even systemic diseases (atherosclerosis, type 2 diabetes), have a pathogenetic hallmark in the so-called dysbiosis [49-54]. The mechanistic link between gut dysbiosis and cardio-

The Interplay between Intestinal and Cardiac Systems in HF vascular impairment, including hypertension, thrombosis, and HF, have gained much attention in recent years, not only for basic research but also for plausible therapeutic implications, still representing a subject for debate [55].

In HF patients, in particular those in a decompensated phase, gut luminal hypoxia and changes in local $\mathrm{pH}$ induced by intestinal hypoperfusion may significantly contribute to a change in the composition of gut microbiota [23]. Several researchers have attempted to characterize the gut microbiota profile in HF, demonstrating significant alterations consistent with bacterial overgrowth, shifting to pathogenetic phyla, and decrease in bacteria with anti-inflammatory functions [31]. Dinakaran et al.[56] showed a significantly higher $16 \mathrm{~S}$ rRNA bacterial copy number in decompensated patients with HF; moreover, in patients with CVDs a dominance of Actinobacteria was found, while Proteobacteria were dominant in controls [55].

Moreover, using 16S rRNA gene amplicon sequencing, different cross-sectional studies reported significant differences between the gut microbial composition of both acutely decompensated and stable HFrEF patients $[31,57,58]$. In stable HF patients, Kummen et al. [57] found a reduction in SCFA butyrate-producing bacteria species, while Pasini et al. [31] found an increase in pathogenic gut bacteria, such as Candida, Campylobacter, Shigella, Salmonella, and Yersinia, as compared to healthy controls. Interestingly, the increase in pathogenic bacteria was correlated with worsening NYHA class [58]. Until now, neither the real composition of microbiota in patients with HF nor the eventual changes occurring during phases of acute decompensation and subsequent stable HF disease are completely clear.

As for other CVDs, the role of bacterial metabolites has been investigated also in HF. Organ et al. [18] showed in mice models that elevated TMAO levels correlated with enhanced susceptibility to develop HF, as evidenced by pathological LV dilatation, reduced LV EF, increased circulating BNP levels, increased pulmonary edema, and increased myocardial fibrosis $[17,18]$. In particular, pulmonary edema, cardiac enlargement, and LV EF were significantly worse in mice fed with TMAO- or cholinesupplemented diet with respect to mice fed with control diet [17-19].

Several mechanisms may contribute to the adverse prognosis in patients with HF and higher TMAO levels; among them are pro-atherogenic properties of TMAO, inflammation, and oxidative stress. High plasma TMAO levels are an independent risk factor for long-term mor- 


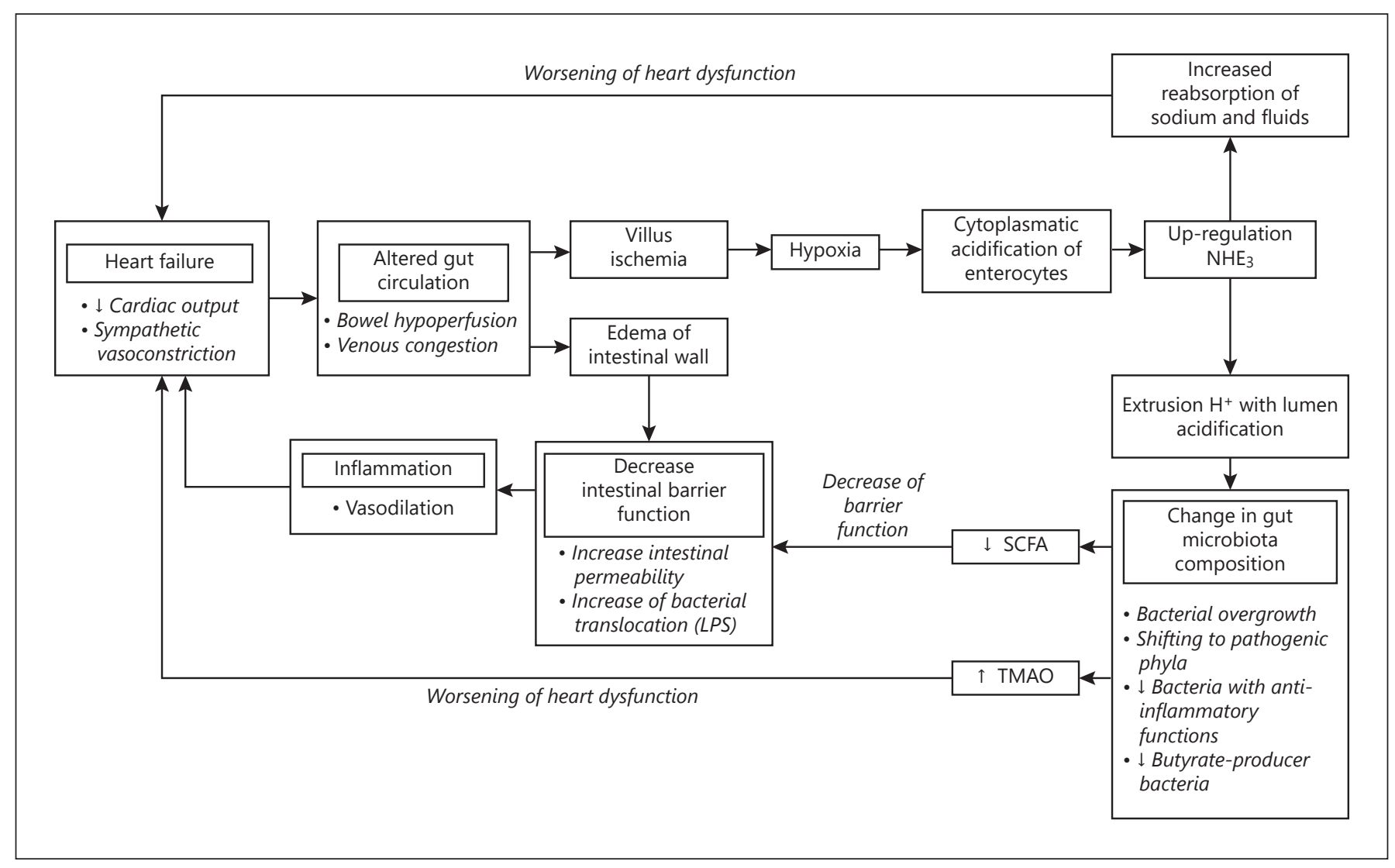

Fig. 1. Pathophysiological mechanisms underlying the associations between HF, splanchnic hemodynamics, gut dysbiosis, and worsening renal and hepatic functions are strongly interrelated. LPS, lipopolysaccharide.

tality in patients with chronic HF, correlating with more advanced LV diastolic dysfunction and poorer long-term adverse clinical outcomes in chronic systolic HF [59]. This correlation is confirmed even after adjusting for natriuretic peptide levels and renal function, underscoring the importance of microbiota-dependent TMAO formation for the prognosis for people with HF, independent of traditional risk factors, including renal dysfunction [59].

In a study on 112 patients with chronic systolic HF, median TMAO levels were significantly higher in subjects with NYHA functional class III or IV. Their levels were also associated with higher plasma N-terminal proBNP (NT-proBNP) levels and more advanced LV diastolic dysfunction. In particular, only higher TMAO levels were associated with a poor prognosis, independently from age, estimated glomerular filtration rate, mitral E/ septal Ea, and NT-proBNP levels [17]. Moreover, the lack of correlations between TMAO, choline, and betaine and other inflammatory biomarkers (plasma myeloperoxi- dase and high-sensitivity CRP) led the authors to suggest an independent pathway for these metabolites in development and progression of HF. Among the mechanisms involved, it was suggested that TMAO may directly impact myocardial fibrosis [19]; moreover, an increase in angiotensin-II mediated hypertensive effects has been reported during direct infusion of TMAO, suggesting the possibility of a neurohormonal synergistic action [16].

Also other bacterial metabolites, such as SCFAs, have been recently known for their potential link with HF. SCFAs represent the main energy source for large intestinal epithelial cells and, through G-protein-coupled receptors, they represent an essential regulator of fluid and electrolyte homeostasis and epithelial barrier maintenance $[60,61]$.

SCFAs secreted by gut flora are normally absorbed by epithelial cells, where they act on the mitochondria stabilizing the hypoxia-inducible factor (HIF), a polypeptide signaling molecule involved in production, and maintenance of several epithelial cell barrier proteins [62]. In 
$\mathrm{HF}$, the hypoxia generated in the gut lumen as a consequence of splanchnic hypoperfusion causes an acidification of the gut lumen and a depletion of butyrate-producing bacteria [63]. Ischemia and relative depletion of SCFAs destabilize HIF, reducing intestinal barrier function, increasing permeability, and leading to low-grade endotoxemia and inflammation. Several lines of evidence also suggest a cardioprotective role of SCFAs, mainly through the promotion of postinfarction cardiac repair through stimulation of CX3CR $1+$ monocytes in the periinfarct zone [64]. Figure 1 summarizes the pathophysiological mechanisms underlying the associations between $\mathrm{HF}$, splanchnic hemodynamic, and gut dysbiosis.

\section{Potential Strategies for the Management of HF}

Based on the mechanistic links between the gut and $\mathrm{HF}$, different alternative strategies have been suggested for the treatment of HF, including modulating the microbiota composition (diet, antibiotics, prebiotics, and probiotics), targeting the biochemical pathway involved in HF pathogenesis by using molecules acting on host/bacterial metabolism and restoring the integrity of intestinal mucosal barrier to prevent bacterial translocation. Interest in this new scenario is growing, although the complexity and the diversity among individuals make the microbiota a very complicated therapeutic target.

\section{Dietary Interventions}

The discovery of dietary-induced gut microbiota-derived metabolites, such as choline, betaine, and TMAO, and their correlation with strong cardiovascular outcomes, led to emphasizing the role of the diet-induced composition of microbiota. The Mediterranean diet, as compared to the "Western world" diet, is rich in fruits, vegetables, legumes, and whole grains, and has been associated with reduced mortality and lower risk of CVDs [65]. Different mechanisms have been suggested to explain the beneficial effects of the Mediterranean diet. In particular, a fiber-rich diet is able to promote an optimal microbial composition, by stimulating the growth of beneficial commensal bacteria and limiting that of known opportunistic bacteria, thus favoring a healthy state and decreasing HF incidence up to $74 \%[66,67]$. A high-fiber diet has been demonstrated to be associated with lower gut dysbiosis, decreased blood pressure, normalized cardiac hypertrophy, and improved cardiac function in an experimental model of hypertension-induced HF [68].

The Interplay between Intestinal and Cardiac Systems in HF
Action on the source of bacterial metabolites known to have an impact on CVDs and HF, such as TMAO, may represent a practicable approach that can be realized by modifying the diet in order to reduce intake of TMA-producing substrates and consequent TMAO production. Choline is a necessary nutrient, therefore, its total elimination by diet is unrealistic, as compared to L-carnitine for instance which is abundant in red meat. Therefore, encouraging to follow a Mediterranean diet, typically low in red meat, may contribute to reduced TMAO levels and consequently favorably impact CVDs [69]. However, litthe is known about the real impact of diet intervention on gut microbial metabolism and CVDs, and strong evidence for the benefit of this strategy in HF is still largely lacking.

\section{Molecular Inhibitors of the TMAO Pathway}

Although dietary intervention still deserves many concerns on its efficacy in HF, interest in the potential role of TMAO as a drug target in this condition is continuously growing. Recent efforts are aimed at developing specific drugs capable of inhibiting the production of TMAO. Konop et al. [70] hypothesized that enalapril, a well-established drug that reduces cardiovascular mortality in HF patients, could modify plasma level of gut bacteria-derived metabolites and the composition of gut microbiota. They found that rats treated with enalapril showed a significantly lower plasma level of TMAO and a trend toward higher 24-h urine excretion of TMA and TMAO [70]. However, there was no significant difference between the groups in the composition of gut bacteria.

It has also been reported that the 3,3-dimethyl-1-butanol, a structural analog of choline, is able to inhibit microbial production of TMA and TMAO, through the inhibition of microbial TMA lyase [71]. A recent study by Wang et al. [72] in rats demonstrated that 3,3-dimethyl1-butanol attenuates pressure overload-induced cardiac remodeling, such as cardiac hypertrophy, fibrosis, and inflammation, by reducing plasma TMAO levels.

Oral administration of specific oral nonabsorbant binders has been suggested to be a promising intervention, by removal of gut microbiota-derived metabolites (e.g., TMAO) or their precursors (e.g., TMA) form the gut [73]. The oral charcoal adsorbent AST-120 has been clinically used to remove uremic toxins from patients with advanced renal failure. In rats with chronic renal disease, AST-120 has been shown to prevent progression of LV hypertrophy and cardiac fibrosis; however, further studies did not confirm the benefit of AST-120 in human HF [23]. 
In general, although these studies confirmed a correlation between elevated blood concentrations of TMAO (or its precursors) and a worse outcome in HF patients, available data do not conclusively support the inhibition of TMAO production as a successful strategy. The heterogeneity of study designs and clinical cohorts, including populations with different stages of disease, global cardiovascular risk, and comorbidities, may explain the weakness of the current results. Moreover, a real cutoff to assess significantly elevated TMAO levels has not been identified yet, and longitudinal evaluation correlating TMAO levels and different phases of HF are still lacking. In conclusion, these interesting approaches are far from being valid alternatives and further studies are needed to investigate the real impact of bacterial metabolites in this field.

\section{Antibiotic Therapy}

Antibiotic therapy may interfere with the pathogenesis of microbiota-driven HF by changing its composition and abundance. Rifaximin, a nonabsorbable oral antibiotic, strongly associated with beneficial effects in cirrhotic patients with hepatic encephalopathy, can reduce endotoxemia and improve hemodynamics by increasing glomerular filtration rate and natriuresis, while reducing plasma endotoxin, IL-6, and TNF-a levels. In 20 patients with decompensated cirrhosis, Kaji et al. [74] found lower plasma endotoxin levels after 4 weeks of treatment with rifaximin $400 \mathrm{mg}$ thrice a day when compared to baseline, but without modifying the overall composition of the gut microbiome. Two possible mechanisms were suggested; the impact of rifaximin on metabolic modifications in the gut microbiota, and the possibility that rifaximin may contribute to the improvement of the intestinal barrier function [74].

The recent gut-heart phase II trial conducted in Norway and Brazil was aimed at assessing the effect, on cardiac function, of the action of gut microbiota in HF patients with reduced $\mathrm{EF}$ treated with rifaximin versus the probiotic yeast Saccharomyces boulardii versus a control group with no treatment. Three months of treatment with both rifaximin and probiotic did not affect cardiac function, which represents the primary endpoint. A significantly change in microbiota composition was found in the rifaximin group after the 3 months of treatment with respect to baseline; however, there was no significant impact on LV EF [75].

Data on other systemic antibiotics and HF are very scarce. Rats treated with vancomycin presented a reduction in myocardial infarct size in an ischemia-reperfusion model [76]. In contrast, direct administration of vancomycin into the coronary circulation did not improve the severity of myocardial infarction. Moreover, the potential side-effects of antibiotics, such as microbial substitution and antibiotic resistance, still challenge the potential role of this strategy and its real impact on HF population.

\section{Probiotics}

Probiotics are live microorganisms able to confer benefits to the host by improving its intestinal balance. It has been suggested that modulation of gut flora by probiotics can favorably modulate cardiac remodeling and function in HF. A small double-blind, placebocontrolled pilot study by Costanza et al. [77] demonstrated in $20 \mathrm{HF}$ patients NYHA (class II or III, with LVEF $<50 \%$ ), randomized to probiotic preparation with Saccharomyces boulardii (1,000 mg per day for 3 months) or placebo, that HF patients treated with a probiotic showed a reduction in uric acid and total cholesterol levels, and an improvement in cardiac systolic function compared with the placebo group [77]. Oral administration of Lactobacillus plantarum in rats has been shown to induce cardioprotective effects, including smaller LV infarct size and improved cardiac function following myocardial ischemia/reperfusion. Similar results were obtained using Lactobacillus rhamnosus GR-1 in a rat model of attenuated LV hypertrophy and improved LV EF. In this study, a decrease in levels of the adipokine leptin was reported and recognized as the potential beneficial probiotic mechanism, while treatment strain did not influence the original gut composition, as revealed by the $16 \mathrm{~S}$ sequencing [78]. However, although probiotic therapy represents a promising and generally safe approach, larger studies as the gut-heart trial did not confirm these results in a 3-month intervention period. Further investigations are needed to determine their contribution in gut microbiota composition and the real long-term benefits of probiotics use in different classes of HF patients.

\section{Fecal Microbiota Transplantation}

Fecal microbiota transplantation (FMT) is a method of treating intestinal microbiological imbalance, mostly used to treat recurrent or refractory Clostridium difficile infections and bowel diseases [79]. Although the efficacy of the application of FMT to other diseases is still poorly known, different studies are examining the potential benefits. Growing evidence suggests that the donor plays an influential role in FMT outcomes for indications outside of CDI. Specifically, in terms of metabolic diseases, 
the efficacy of FMT would depend on the ability of the donor to provide the necessary taxa capable of restoring metabolic deficits in recipients that are contributing toward the disease [80]. Until now, only a study on mice showed that gut microbial transplantation can transmit choline diet-induced TMAO production and atherosclerosis susceptibility. In particular, microbial DNA analyses in feces and cecum revealed that transplantation of donor microbial community features into recipients also correlated with plasma TMAO levels in donors and atherosclerosis susceptibility [81]. It may be possible to reduce TMAO in patients with HF by transplanting low TMAO-producing strains, but this clearly requires more studies.

\section{Inhibition of NHE3}

As mentioned above, members of the sodium/hydrogen exchanger (NHE) family of proteins, which are expressed throughout the gastrointestinal tract, facilitate the electroneutral exchange of sodium ions for intracellular hydrogen ions across membranes. NHE3 is particularly important for intestinal sodium transport. Tenapanor is a minimally absorbed small molecule representing an inhibitor of NHE3 that acts locally in the gastrointestinal tract [82]. In animals and human recent studies, tenapanor has been reported to reduce absorption of sodium and phosphate from the gut, with a concomitant increase in stool fluid content. In particular, tenapanor treatment over 7 days reduced sodium absorption, resulting in increases in stool sodium content of up to $50 \mathrm{mmol}$ ( $2.9 \mathrm{~g}$ table salt) per day [73]. In patients with HF, usually characterized by fluid overload, tenapanor has been suggested as a reliable strategy, mainly in patients developing resistance to diuretics and/or developing concomitant renal failure and hypokalemia. Drugs acting in reducing intestinal sodium absorption, like tenapanor, play a relevant role as antihypertensive agents, with a significant impact on the most common risk factor for CVDs.

In addition to increased stool sodium excretion, tenapanor has also been found to increase the excretion of stool phosphate and reduce serum phosphate concentrations which have beneficial implications for patients with CKD-related hyperphosphatemia [83]. Moreover, tenapanor is in general reported to be well tolerated in humans with only mild to moderate adverse GI effects such as abdominal pain and diarrhea. The role of gastrointestinal NHE3 inhibition in treating HF, therefore, represents an interesting approach, requiring further investigation.

The Interplay between Intestinal and Cardiac Systems in HF

\section{Conclusions}

HF still remains a major health burden; with the prolongation of life expectancy, cardiac diseases currently continue to escalate. Although several treatment regimens are available, their effectiveness is often unsatisfactory. There is growing evidence that gut microbiota disorders, intestinal barrier dysfunction, and gut microbiota metabolites have an impact on CVDs. Although the interactions between gut and heart are very complex (and, still unclear), a close mechanistic link seems evident leading to the definition of "Gut Hypothesis of HF."

We have come a long way since the first pioneering researches correlating TMA/TMAO pathway with the atherosclerosis; metagenomic and metabolomic studies have highlighted increasingly refined links. 16S-based studies in HF patients found a significant reduction in SCFAs-producer bacteria, demonstrating their impact on the integrity of the intestinal mucosa and barrier dysfunction. Another great part of these studies focused on microbiota-derived metabolites, such as TMAO and LPS, suggesting clinical implications in terms of inflammatory response modulation, molecular inhibitors, and key mediator molecules.

Of course, when we talk about microbiota we refer to an increasingly characterized system, which is still in an infant state and of which little is known. Gut microbiota is now recognized as a microbial ecosystem more complex than previously imagined, and our understanding of the links between changes in the microbiota and diseases is constantly evolving.

Different studies reported significantly differences in gut bacterial composition between HF patients and healthy controls; however, identification of a common microbiome related to HF has not been recognized yet. The great heterogeneity of HF population, both in the real word and in clinical trials, has also to deal with influence of common factors influencing microbiota composition like diet and medications. Therefore, despite encouraging results, therapeutic strategies based on microbiota modulation are still far from being validated in clinical practice.

In more recent days, promising results came from studies on NH3 inhibitors, for their capacity to reduce sodium overload; in particular, this could be a very reliable strategy in HF refractory to diuretics or in patients with concomitant renal failure. Larger studies are needed to fully understand the strong associations between microbiota composition, their functions, and their influence on the heart, in order to translate the research to successful therapeutic interventions.

Med Princ Pract 2022;31:203-214 


\section{Conflict of Interest Statement}

The authors have no conflicts of interest to declare.

\section{Funding Sources}

This study received no grant from any funding agency.

\section{Author Contributions}

Antonella Gallo and Massimo Montalto: study concept and design; Noemi Macerola, Antonella Gallo, and Maria Anna Nicolazzi: drafting of the manuscript. All authors contributed to literature review, interpretation of data, and critical revision of the manuscript. All authors approved the final version of the manuscript.

\section{References}

1 Writing Group Members; Mozaffarian D, Benjamin EJ, Go AS, Arnett DK, Blaha MJ, et al. Heart disease and stroke statistics-2016 update. Circulation. 2016;133. e38-e360

2 Gutman SJ, Costello BT, Papapostolou S, Iles L, Ja J, Hare JL, et al. Impact of sex, socio-economic status, and remoteness on therapy and survival in heart failure. ESC Heart Fail. 2019;6:944-52.

3 Tanai E, Frantz S. Pathophysiology of heart failure. Compr Physiol. 2016;6:187-214.

4 Sandek A, Doehner W, Anker SD, von Haehling S. Nutrition in heart failure: an update. Curr Opin Clin Nutr Metab Care. 2009;12:384-91.

5 Rogler G, Rosano G. The heart and the gut. Eur Heart J. 2014;35:426-30.

6 Ferreira CM, Vieira AT, Vinolo MA, Oliveira FA, Curi R, Martins Fdos S, et al. The central role of the gut microbiota in chronic inflammatory diseases. J Immunol Res. 2014;2014:689492.

7 Qin J, Li R, Raes J, Arumugam M, Burgdorf KS, Manichanh C, et al. A human gut microbial gene catalogue established by metagenomic sequencing. Nature. 2010; 464:59-65.

8 Jones ML, Ganopolsky JG, Martoni CJ, Labbé A, Prakash S. Emerging science of the human microbiome. Gut Microbes. 2014;5:446-57.

9 Neish AS. Microbes in gastrointestinal health and disease. Gastroenterology. 2009; 136(1):65-80.

10 Weiss GA, Hennet T. Mechanisms and consequences of intestinal dysbiosis. Cell Mol Life Sci. 2017;74:2959-77.

11 Goodrich JK, Waters JL, Poole AC, Sutter JL, Koren O, Blekhman R, et al. Human genetics shape the gut microbiome. Cell. 2014;159:789-99.

12 Wang Z, Klipfell E, Bennett BJ, Koeth R, Levison BS, Dugar B, et al. Gut flora metabolism of phosphatidylcholine promotes cardiovascular disease. Nature. 2011;472: 57-63.

13 Tang WH, Wang Z, Levison BS, Koeth RA, Britt $\mathrm{EB}, \mathrm{Fu} \mathrm{X}$, et al. Intestinal microbial metabolism of phosphatidylcholine and cardiovascular risk. N Engl J Med. 2013; 368:1575-84
14 Koeth RA, Wang Z, Levison BS, Buffa JA, Org E, Sheehy BT, et al. Intestinal microbiota metabolism of l-carnitine, a nutrient in red meat, promotes atherosclerosis. Nat Med. 2013;19:576-85.

15 Wang Z, Tang WH, Buffa JA, Fu X, Britt EB, Koeth RA, et al. Prognostic value of choline and betaine depends on intestinal microbiota-generated metabolite trimethylamine-N-oxide. Eur Heart J. 2014;35: 904-10.

16 Ufnal M, Jazwiec R, Dadlez M, Drapala A Sikora M, Skrzypecki J. TrimethylamineN-oxide: a carnitine-derived metabolite that prolongs the hypertensive effect of angiotensin II in rats. Can J Cardiol. 2014;30: 1700-5.

17 Tang WH, Wang Z, Shrestha K, Borowski AG, Wu Y, Troughton RW, et al. Intestinal microbiota-dependent phosphatidylcholine metabolites, diastolic dysfunction, and adverse clinical outcomes in chronic systolic heart failure. J Card Fail. 2015;21:91-6.

18 Organ CL, Otsuka H, Bhushan S, Wang Z, Bradley J, Trivedi R, et al. Choline diet and its gut microbe-derived metabolite, trimethylamine $\mathrm{N}$-oxide, exacerbate pressure overload-induced heart failure. Circ Hear Fail. 2016;9:e002314.

19 Li Z, Wu Z, Yan J, Liu H, Liu Q, Deng Y, et al. Gut microbe-derived metabolite trimethylamine $\mathrm{N}$-oxide induces cardiac hypertrophy and fibrosis. Lab Investig. 2019; 99:356-7.

20 Heianza Y, Ma W, Manson JE, Rexrode KM, Qi L. Gut microbiota metabolites and risk of major adverse cardiovascular disease events and death: a systematic review and meta-analysis of prospective studies. J Am Heart Assoc. 2017;6:e004947.

21 Ponikowski P, Voors AA, Anker SD, Bueno $\mathrm{H}$, Cleland JGF, Coats AJS, et al. 2016 ESC guidelines for the diagnosis and treatment of acute and chronic heart failure. Eur Heart J. 2016;37:2129-200.

22 Tang WH, Wang Z, Fan Y, Levison B, Hazen JE, Donahue LM, et al. Prognostic value of elevated levels of intestinal microbegenerated metabolite trimethylamine- $\mathrm{N}$ oxide in patients with heart failure: refining the gut hypothesis. J Am Coll Cardiol. 2014;64:1908-14.
23 Nagatomo Y, Tang WH. Intersections between microbiome and heart failure: revisiting the gut hypothesis. J Card Fail. 2015; 21:973-80

24 Tromp J, Teng TH, Tay WT, Hung CL, Narasimhan C, Shimizu W, et al. Heart failure with preserved ejection fraction in Asia. Eur J Heart Fail. 2019;21:23-36.

25 Tedeschi A, Agostoni P, Pezzuto B, Corra U, Scrutinio D, La Gioia R, et al. Role of comorbidities in heart failure prognosis part 2: chronic kidney disease, elevated serum uric acid. Eur J Prev Cardiol. 2020;27: $35-45$.

26 Bourlaug B. Evaluation and management of heart failure with preserved ejection fraction. Nat Rev Cardiol. 2020;17:559-73.

27 Madamanchi C, Alhosaini H, Sumida A, Runge MS. Obesity and natriuretic peptides, BNP and NT-proBNP: mechanisms and diagnostic implications for heart failure. Int J cardiol. 2014;176:611-7.

28 Brown JM, Hazen SL. The gut microbial endocrine organ: bacterially derived signals driving cardiometabolic diseases. Annu Rev Med. 2015;66:343-59.

29 Frangogiannis NG. The inflammatory response in myocardial injury, repair, and remodelling. Nat Rev Cardiol. 2014;11:25565.

30 Tang WHW, Li DY, Hazen SL. Dietary metabolism, the gut microbiome, and heart failure. Nat Rev Cardiol. 2019;16:137-54

31 Pasini E, Aquilani R, Testa C, Baiardi P, Angioletti S, Boschi F, et al. Pathogenic gut flora in patients with chronic heart failure. JACC Hear Fail. 2016;4:220-7.

32 Kalogeropoulos AP, Tang WH, Hsu A, Felker GM, Hernandez AF, Troughton RW, et al. High-sensitivity C-reactive protein in acute heart failure: insights from the ASCEND-HF trial. J Card Fail. 2014;20: 319-26.

33 Topf A, Mirna M, Ohnewein B, Jirak P, Kopp K, Fejzic D, et al. The diagnostic and therapeutic value of multimarker analysis in heart failure. An approach to biomarkertargeted therapy. Front Cardiovascular Med. 2020;7:570567.

Gallo/Macerola/Favuzzi/Nicolazzi/ Gasbarrini/Montalto 
34 Mogensen UM, Gong J, Jhund PS, Shen L, Køber L, Desai AS, et al. Effect of sacubitril/ valsartan on recurrent events in the prospective comparison of ARNI with ACEI to determine impact on global mortality and morbidity in heart failure trial (PARADIGM-HF). Eur J Heart Fail. 2018;20:7608.

35 Butler J, Hamo CE, Filippatos G, Pocock SJ, Bernstein RA, Brueckmann M, et al. The potential role and rationale for treatment of heart failure with sodiumglucose cotransporter 2 inhibitors. Eur J Heart Fail. 2017;19:1390-400.

36 Polsinelli VB, Sinha A, Shah SJ. Visceral congestion in heart failure: right ventricular dysfunction, splanchnic hemodynamics, and the intestinal microenvironment. Curr Heart Fail Rep. 2017;14:519-28.

37 Sandek A, Bauditz J, Swidsinski A, Buhner S, Weber-Eibel J, von Haehling S, et al. Altered intestinal function in patients with chronic heart failure. J Am Coll Cardiol. 2007;50:1561-9.

38 Sandek A, Swidsinski A, Schroedl W, Watson A, Valentova M, Herrmann R, et al. Intestinal blood flow in patients with chronic heart failure: a link with bacterial growth, gastrointestinal symptoms, and cachexia. J Am Coll Cardiol. 2014;64:1092-102.

39 Stillhart C, Vučićević K, Augustijns P, Basit AW, Batchelor H, Flanagan TR, et al. Impact of gastrointestinal physiology on drug absorption in special populations: an UNGAP review. Eur J Pharm Sci. 2020;147: 105280.

40 Bosch L, Lam CSP, Gong L, Chan SP, Sim $\mathrm{D}$, Yeo $\mathrm{D}$, et al. Right ventricular dysfunction in left-sided heart failure with preserved versus reduced ejection fraction. Eur J Heart Fail. 2017;19:1664-71.

41 Mullens W, Abrahams Z, Francis GS, Sokos G, Taylor DO, Starling RC, et al. Importance of venous congestion for worsening of renal function in advanced decompensated heart failure. J Am Coll Cardiol. 2009; 53:589-96.

42 Valentova $\mathrm{M}$, von Haehling S, Bauditz J, Doehner W, Ebner N, Bekfani T, et al. Intestinal congestion and right ventricular dysfunction: a link with appetite loss, inflammation, and cachexia in chronic heart failure. Eur Heart J. 2016;37:1684-91.

43 Rossi M, Sganga G, Mazzone M, Valenza V, Guarneri S, Portale G, et al. Cardiopulmonary bypass in man: role of the intestine in a self-limiting inflammatory response with demonstrable bacterial translocation. Ann Thorac Surg. 2004;77:612-8.

44 Linz B, Hohl M, Mishima R, Saljic A, Lau $\mathrm{DH}$, Jespersen T, et al. Pharmacological inhibition of sodium-proton-exchanger subtype 3-mediated sodium absorption in the gut reduces atrial fibrillation susceptibility in obese spontaneously hypertensive rats. Int J Cardiol Heart Vasc. 2020;28:100534.
45 He P, Yun CC. Mechanisms of the regulation of the intestinal $\mathrm{Na}+\mathrm{H}+$ exchanger NHE3. J Biomed Biotechnol. 2010;2010: 238080.

46 Furness JB, Rivera LR, Cho HJ, Bravo DM, Callaghan B. The gut as a sensory organ. Nat Rev Gastroenterol Hepatol. 2013;10: 729-40.

47 Harrison CA, Laubitz D, Ohland CL, Midura-Kiela MT, Patil K, Besselsen DG, et al. Microbial dysbiosis associated with impaired intestinal $\mathrm{Na}+/ \mathrm{H}+$ exchange accelerates and exacerbates colitis in ex-germ free mice. Mucosal Immunol. 2018;11: 1329-41.

48 Arumugam M, Raes J, Pelletier E, Le Paslier D, Yamada T, Mende DR, et al. Enterotypes of the human gut microbiome. Nature. 2011;473:174-80.

49 Feld JJ, Meddings J, Heathcote EJ. Abnormal intestinal permeability in primary biliary cirrhosis. Dig Dis Sci. 2006;51:1607-13.

50 Assimakopoulos SF, Scopa CD, Vagianos CE. Pathophysiology of increased intestinal permeability in obstructive jaundice. World J Gastroenterol. 2007;13:6458-64.

51 Vaziri ND, Yuan J, Norris K. Role of urea in intestinal barrier dysfunction and disruption of epithelial tight junction in chronic kidney disease. Am J Nephrol. 2013;37:1-6.

52 Rosenfeld CS. Microbiome disturbances and autism spectrum disorders. Drug Metab Dispos. 2015;43:1557-71.

53 Sun MF, Shen YQ. Dysbiosis of gut microbiota and microbial metabolites in Parkinson's disease. Ageing Res Rev. 2018;45:5361.

54 Dabke K, Hendrick G, Devkota S. The gut microbiome and metabolic syndrome. J Clin Invest. 2019;129:4050-7.

55 Tang WH, Hazen SL. The contributory role of gut microbiota in cardiovascular disease. J Clin Invest. 2014;124:4204-11.

56 Dinakaran V, Andiappan R, Muthuirulan P, Ramamoorthy S, Paramasamy G, Jeyaprakash R. Elevated levels of circulating DNA in cardiovascular disease patients: metagenomic profiling of microbiome in the circulation. Plos One 2014 Aug 18;9(8): e105221.

57 Kummen M, Mayerhofer CCK, Vestad B, Broch K, Awoyemi A, Storm-Larsen C, et al. Gut microbiota signature in heart failure defined from profiling of 2 independent cohorts. J Am Coll Cardiol. 2018;71:11846.

58 Madan S, Mehra MR. Gut dysbiosis and heart failure: navigating the universe within. Eur J Heart Fail. 2020;22:629-37.

59 Li W, Huang A, Zhu H, Liu X, Huang X, Huang $Y$, et al. Gut microbiota-derived trimethylamine $\mathrm{N}$-oxide is associated with poor prognosis in patients with heart failure. Med J Aust. 2020;213:374-9.
60 Shimizu H, Masujima Y, Ushiroda C, Mizushima R, Taira S, Ohue-Kitano R, et al. Dietary short-chain fatty acid intake improves the hepatic metabolic condition via FFAR3. Sci Rep. 2019;9:16574.

61 Brown AJ, Goldsworthy SM, Barnes AA, Eilert MM, Tcheang L, Daniels D, et al. The orphan G protein-coupled receptors GPR41 and GPR43 are activated by propionate and other short chain carboxylic acids. J Biol Chem. 2003;278:11312-9.

62 Kelly CJ, Zheng L, Campbell EL, Saeedi B, Scholz CC, Bayless AJ, et al. Crosstalk between microbiota-derived short-chain fatty acids and intestinal epithelial HIF augments tissue barrier function. Cell Host Microbe. 2015;17:662-71.

63 Cui X, Ye L, Li J, Jin L, Wang W, Li S, et al. Metagenomic and metabolomic analyses unveil dysbiosis of gut microbiota in chronic heart failure patients. Sci Rep. 2018;8:635.

64 Tang TWH, Chen HC, Chen CY, Yen CYT, Lin CJ, Prajnamitra RP, et al. Loss of gut microbiota alters immune system composition and cripples postinfarction cardiac repair. Circulation. 2019;139(5):647-59.

65 Widmer RJ, Flammer AJ, Lerman LO, Lerman A. The mediterranean diet, its components, and cardiovascular disease. Am J Med. 2015;128:229-38.

66 Branchereau M, Burcelin R, Heymes C. The gut microbiome and heart failure: a better gut for a better heart. Rev Endocr Metab Disord. 2019;20:407-14.

67 Lopez-Garcia E, Rodriguez-Artalejo F, Li TY, Fung TT, Li S, Willett WC, et al. The mediterranean-style dietary pattern and mortality among men and women with cardiovascular disease. Am J Clin Nutr. 2014; 99:172-80.

68 Marques FZ, Nelson E, Chu PY, Horlock D, Fiedler A, Ziemann M, et al. High-fiber diet and acetate supplementation change the gut microbiota and prevent the development of hypertension and heart failure in hypertensive mice. Circulation. 2017;135: 964-77.

69 Zabell A, Tang WH. Targeting the microbiome in heart failure. Curr Treat Options Cardiovasc Med. 2017;19:27.

70 Konop M, Radkowski M, Grochowska M, Perlejewski K, Samborowska E, Ufnal M, et al. Enalapril decreases rat plasma concentration of TMAO, gut bacteria-derived cardiovascular marker. Biomarkers. 2018;23: 380-5.

71 Wang Z, Roberts AB, Buffa JA, Levison BS, Zhu W, Org E, et al. Non-lethal inhibition of gut microbial trimethylamine production for the treatment of atherosclerosis. Cell. 2015;163:1585-95.

72 Wang G, Kong B, Shuai W, Fu H, Jiang X, Huang H. 3,3-Dimethyl-1-butanol attenuates cardiac remodeling in pressure-overload-induced heart failure mice. J Nutr Biochem. 2020;78:108341.
The Interplay between Intestinal and Cardiac Systems in HF
Med Princ Pract 2022;31:203-214 
73 Fujii H, Nishijima F, Goto S, Sugano M, Yamato H, Kitazawa R, et al. Oral charcoal adsorbent (AST-120) prevents progression of cardiac damage in chronic kidney disease through suppression of oxidative stress. Nephrol Dial Transplant. 2009;24: 2090-5.

74 Kaji K, Takaya H, Saikawa S, Furukawa M, Sato S, Kawaratani H, et al. Rifaximin ameliorates hepatic encephalopathy and endotoxemia without affecting the gut microbiome diversity. World J Gastroenterol. 2017;23:8355-66.

75 Awoyemi A, Mayerhofer C, Felix AS, Hov JR, Moscavitch SD, Lappegård KT, et al. Rifaximin or saccharomyces boulardii in heart failure with reduced ejection fraction: results from the randomized gut heart trial. EBioMedicine. 2021 Aug;70:103511.
76 Cannon $\mathrm{CP}$, Braunwald E, McCabe $\mathrm{CH}$, Grayston JT, Muhlestein B, Giugliano RP, et al. Antibiotic treatment of chlamydia pneumoniae after acute coronary syndrome. N Engl J Med. 2005;352:1646-54.

77 Costanza AC, Moscavitch SD, Faria Neto HC, Mesquita ET. Probiotic therapy with Saccharomyces boulardii for heart failure patients: a randomized, double-blind, placebo-controlled pilot trial. Int J Cardiol. 2015;179:348-50.

78 Gan XT, Ettinger G, Huang CX, Burton JP, Haist JV, Rajapurohitam V, et al. Probiotic administration attenuates myocardial hypertrophy and heart failure after myocardial infarction in the rat. Circ Hear Fail. 2014;7:491-9.

79 Cammarota G, Gallo A, Ianiro G, Montalto M. Emerging drugs for the treatment of clostridium difficile. Expert Opin Emerg Drugs. 2019;24:17-28.
80 Wilson BC, Vatanen T, Cutfield WS, O'Sullivan JM. The super-donor phenomenon in fecal microbiota transplantation. Front Cell Infect Microbiol. 2019;9:2.

81 Gregory JC, Buffa JA, Org E, Wang Z, Levison $\mathrm{BS}$, $\mathrm{Zhu} \mathrm{W}$, et al. Transmission of atherosclerosis susceptibility with gut microbial transplantation. J Biol Chem. 2015; 290:5647-60.

82 King AJ, Siegel M, He Y, Nie B, Wang J, Koo-McCoy S, et al. Inhibition of sodium/ hydrogen exchanger 3 in the gastrointestinal tract by tenapanor reduces paracellular phosphate permeability. Sci Transl Med. 2018;10:eaam6474.

83 Johansson S, Rosenbaum DP, Knutsson M, Leonsson-Zachrisson M. A phase 1 study of the safety, tolerability, pharmacodynamics, and pharmacokinetics of tenapanor in healthy Japanese volunteers. Clin Exp Nephrol. 2017;21:407-16. 